\title{
Evolution of a Viscous Protoplanetary Disk with Convectively Unstable Regions
}

\author{
Ya. N. Pavlyuchenkov, ${ }^{1, *} \quad$ A. V. Tutukov, ${ }^{1}$ \\ L. A. Maksimova, ${ }^{1}$ and E. I. Vorobyov $^{2,3}$ \\ ${ }^{1}$ Institute of Astronomy, RAS, Moscow, Russia \\ ${ }^{2}$ Southern Federal University, Institute of Physics, Rostov-on-Don, Russia \\ ${ }^{3}$ Department of Astrophysics, Vienna University, Vienna, Austria
}

\begin{abstract}
The role of convection in the gas-dust accretion disk around a young star is studied. The evolution of a Keplerian disk is modeled using the Pringle equation, which describes the time variations of the surface density under the action of turbulent viscosity. The distributions of the density and temperature in the polar directions are computed simultaneously in the approximation that the disk is hydrostatically stable. The computations of the vertical structure of the disk take into account heating by stellar radiation, interstellar radiation, and viscous heating. The main factor governing evolution of the disk in this model is the dependence of the viscosity coefficient on the radius of the disk. The computations of this coefficient take into account the background viscosity providing the continuous accretion of the gas and the convective viscosity, which depends on the parameters of the convection at a given radius. The results of computations of the global evolution and morphology of the disk obtained in this approach are presented. It is shown that, in the adopted model, the accretion has burst-like character: after the inner part of the disk ( $\mathrm{R}$ $<3 \mathrm{AU})$ is filled with matter, this material is relatively fast discharged onto the star, after which the process is repeated. Our results may be useful for explaining the activity of young FU Ori and EX Lup objects. It is concluded that convection may be one of the mechanisms responsible for the non-steady pattern of accretion in protostellar disks.
\end{abstract}

* Electronic address: pavyar@inasan.ru 


\section{INTRODUCTION}

Studies of protoplanetary disks of young stars are of considerable interest. A young star accumulates most of its mass via the accretion of gas from a circumstellar disk; i.e., the formation of the star has a direct connection to the evolution of the disk. In turn, a planetary system is a natural result of the late stages of the evolution of the protostellar disk. An enormous number of studies have been devoted to the physics and evolution of protoplanetary disks (see, e.g., the monograph [1]). Despite great observational and theoretical achievements in the study of protoplanetary disks, many problems related to the physics of accretion disks have not been resolved completely. In particular, the question of the mechanism for mass and angular momentum transfer, which enables the accretion of the ambient gas, remains topical. The main approach to describing disk accretion, along with gravitational instability, is the formalism of turbulent viscosity, but the mechanism making the flow turbulent remains under discussion. The convection of accreted gas in the polar directions may be one of the mechanisms giving rise to the turbulence.

The idea that convection in the protoplanetary disks may be responsible not only for heat transfer, but also providing viscosity, and, thus, may influence the evolution of the disk, was formulated in the 1980s [2,3]. This idea was greeted with enthusiasm, however, even after several decades, the role of convection in the transfer of angular momentum remains under discussion (see the detailed historical review [4]). The first numerical models that took convection into account $[5,6]$ provided evidence that the viscosity coefficient corresponding to the convection is very small, and that the gaseous disk itself tends to break up into rings. Studies based on later numerical models $[7,8]$ indicated higher viscosity coefficients (in terms of the Shakura-Sunyaev parameter $\alpha=10^{-3}-10^{-2}$ ), however, this required an additional mechanism for heating the gas in the equatorial plane of the accretion disk. The idea of additional viscosity associated with convection in circumstellar disks was also discussed in [9-11]. When high-quality images of protoplanetary disks were obtained and ring-shaped structures were observed, interest in hydrodynamical models with convection again increased. For example, the results of three-dimensional modeling of convection in a disk were presented in [12]. This demonstrated the rise of convective cells, vortices, and other coherent structures when convection was triggered. Held and Letter [12] note that they were unable to obtain a self-sustaining convection regime in the disk. At the same time, 
theoretical analyses of the convective instability of the accretion disk were presented [13-15], where the instability conditions were found and the need for more detailed models was noted. In the present study, we have investigated the conditions for the rise of convection and the large-scale evolution of a convective protostellar Keplerian disk, based on a model featuring a detailed calculation of the vertical structure of the disk and allowance for the constant accretion of gas onto the disk from a circumstellar envelope. Our main focus is the analysis of the recursive nature of the accretion activity of the protostellar disk.

\section{THE MODEL OF THE DISK}

The evolution of the circumstellar disk was computed using a numerical model in which the evolution of the radial and vertical structure of the disk is calculated consequentially. Each time step is divided into two stages: (i) computation of the evolution of the surface density of the gas; (ii) reconstruction of the density and temperature distributions in the vertical direction. In the first stage, the rate of heating of the medium associated with the accretion of matter, which is necessary for computing the thermal structure, was also computed. Once the vertical structure of the disk was reconstructed, convectively unstable regions were identified and the distribution of the viscosity coefficient needed to compute the evolution of the surface density was determined. We describe both stages of our computations in more detail below.

\subsection{Computation of the Evolution of the Surface Density}

To describe the evolution of the surface density of the disk, we used the formalism of a viscous accretion disk. This approach assumes that the mechanism for the transfer of mass and angular momentum is some physical process (turbulence, magnetic fields, convection), mathematically described in a way analogous to a molecular viscosity; i.e., the model is based on the Navier-Stokes equations. In the approximation of an axially symmetric, geometrically thin Keplerian disk, neglecting gas pressure gradients in the radial direction, the evolution of the surface density is described by the Pringle equation [16]:

$$
\frac{\partial \Sigma}{\partial t}=\frac{3}{R} \frac{\partial}{\partial R}\left[\sqrt{R} \frac{\partial}{\partial R}(\nu \sqrt{R} \Sigma)\right]+W(R, t),
$$


where $\Sigma$ is the surface density, $R$ the distance to the star, $t$ time, $\nu$ the coefficient of kinematic viscosity, and $W(R, t)$ the rate of matter inflow from the envelope. The Pringle equation is widely used to describe the long-term evolution of circumstellar disks (see, e.g., the review [17]). The specifics of its application depend on the definition of the function $\nu(R)$. In our case, $\nu(R)$ is determined phenomenologically and using the conditions of convective instability.

As a result of the evolution of a viscous disk, thermal energy is released. In essence, the source of the released thermal energy is the gravitational energy of the matter; i.e., viscosity may be considered as a mechanism that transforms the gravitational energy of the accreting gas into heat. The rate of heat release per unit area is [16]:

$$
\Gamma_{\text {vis }}=\frac{9}{4} \frac{G M_{\odot}}{R^{3}} \nu \Sigma,
$$

where $M_{\odot}$ is the mass of the central star. This heating was included in the computations of the vertical disk structure. In essence, it is precisely this heating that is responsible for the rise of convection.

\subsubsection{Viscosity coefficient in convectively stable regions}

We assumed the presence of some background mechanism for the transfer of angular momentum in convectively stable regions and specified the viscosity in the form:

$$
\nu_{\mathrm{bg}}=\nu_{0}\left(\frac{R}{R_{\mathrm{AU}}}\right)^{\beta},
$$

where $\beta=1$ and $\nu_{0}=10^{15} \mathrm{~cm}^{2} / \mathrm{s}$. With this choice of $\beta$, the surface density distribution for the stationary solution of the Pringle equation at $R \gg R_{\odot}$,

$$
\dot{M}=3 \pi \Sigma \cdot \nu_{\mathrm{bg}},
$$

will be inversely proportional to the distance to the star, i.e., $\Sigma \propto R^{-1}$, in good agreement with observations of protoplanetary disks [18]. For the adopted value of $\nu_{0}$ and the accretion rate $\dot{M}=10^{-7} M_{\odot} /$ yr, the mass of the disk between 0.1 and $100 \mathrm{AU}$ will be $M_{\text {disk }}=10^{-1} M_{\odot}$. These values also fit the observed ranges of accretion rates and masses of protoplanetary disks [19]. We will now show that the values $\beta=1$ and $\nu_{0}=10^{15} \mathrm{~cm}^{2} / \mathrm{s}$ are consistent with the widely used $\alpha$-parameterization of the turbulent viscosity. According to Shakura 
and Sunyaev [20], $\nu=\alpha \mathrm{c}_{s} H$, where $c_{s}$ is the sound speed and $H$ the height of the disk. Let thermal structure of the disk be completely determined by heating by the central star. Then,

$$
\varepsilon \frac{L \odot}{4 \pi R^{2}}=\sigma T^{4}
$$

where $L_{\odot}$ is the stellar luminosity, $\varepsilon$ the cosine of the angle between the normal to the surface of the disk and the direction to the star, $\sigma$ the Stefan-Boltzmann constant, and $T$ the effective temperature. The condition of hydrostatic equilibrium of the disk in the vertical direction can be approximated

$$
\frac{H}{R} \approx \frac{c_{s}}{V_{k}}
$$

where $V_{k}$ is the Keplerian velocity at the radius $R$. Combining these two equations and using the relation $c_{s}^{2}=k_{\mathrm{B}} T / \mu_{\mathrm{g}} m_{\mathrm{H}}$, where $k_{\mathrm{B}}$ is Boltzmann's constant, $\mu_{\mathrm{g}}$ the mean molecular weight of the matter, and $m_{\mathrm{H}}$ the mass of a hydrogen atom, we obtain:

$$
\nu_{\mathrm{bg}}=\alpha \nu_{\alpha}\left(\frac{R}{R_{\mathrm{AU}}}\right), \text { where } \nu_{\alpha}=\frac{k_{\mathrm{B}} R_{\mathrm{AU}}}{\mu_{\mathrm{g}} m_{\mathrm{H}}\left(G M_{\odot}\right)^{1 / 2}}\left(\frac{\varepsilon L_{\odot}}{4 \pi \sigma}\right)^{1 / 4}
$$

Assuming $\varepsilon=0.1$ and inserting the values of the constants, we obtain $\nu_{\alpha}=10^{16} \mathrm{~cm}^{2} / \mathrm{s}$. Thus, the parameterization (3) we have used for convectively stable regions corresponds to a viscous-disk model with $\alpha=0.1$. This high value of $\alpha$ corresponds to the earliest (less than $1 \mathrm{Myr}$ ) stages of the evolution of protoplanetary disks, when the main contribution to the viscosity is probably made by the self-gravity of the disk [21].

\subsubsection{Coefficient of viscosity in convectively unstable regions}

We will now describe the algorithm we used to specify the viscosity in convectively unstable regions. After computing the vertical structure of the disk using the method described in Section 2.2, the regions of the disk in which the condition of convective instability was satisfied were identified. An area was considered to be convectively unstable if the following condition was satisfied [22]:

$$
\frac{d T}{d z}<-\frac{g(z)}{c_{P}}
$$

where $g(z)=\frac{G M_{\odot}}{R^{3}} z+4 \pi G \sigma(z)$ is the vertical component of the gravitational acceleration at the radial distance $R$ and height $z, \sigma(z)=\int_{0}^{z} \rho\left(z^{\prime}\right) d z^{\prime}$ the surface density measured from 
the equator, $c_{P}=\frac{7}{2} \frac{k_{\mathrm{B}}}{\mu_{\mathrm{g}} m_{\mathrm{H}}}$ the gas specific heat at constant pressure, and $\mu_{\mathrm{g}}=2.4$ the mean molecular weight. The criterion (8) corresponds to restrictions lying at the basis of the model used to reconstruct the vertical structure. In particular, this model does not take into account ionization and dissociation of the gas. Further, for every vertical column defined by the radial coordinate $R$, we computed the mass fraction of the gas $\gamma$ in convectively unstable regions in this column. The viscosity coefficient determined by convection is

$$
\nu_{\mathrm{c}}=\gamma H V_{\mathrm{c}}
$$

where $H$ is the local disk scale height, determined using Eq. (6), and $V_{\mathrm{c}}$ the characteristic convection speed. The speed $V_{\mathrm{c}}$ was determined in the approximation that all the energy released by the accretion of gas is transformed into kinetic energy of the gas' convective motion; i.e., the rate of viscous dissipation is equal to the kinetic energy flux:

$$
\Gamma_{\mathrm{vis}}=\frac{\rho_{0} V_{\mathrm{c}}^{2}}{2} V_{\mathrm{c}}
$$

where $\rho_{0}$ is the equatorial density. The resulting distribution was smoothed in the radial direction using a Gaussian with width $H$ :

$$
\tilde{\nu}_{\mathrm{c}}(R)=\frac{\int_{R_{\text {in }}}^{R_{\text {out }}} \nu_{\mathrm{c}}(r) \mathrm{e}^{-\frac{(R-r)^{2}}{2 H^{2}}} d r}{\int_{R_{\text {in }}}^{R_{\text {out }}} \mathrm{e}^{-\frac{(R-r)^{2}}{2 H^{2}}} d r},
$$

where $R_{\text {in }}$ and $R_{\text {out }}$ are the inner and outer boundaries of the disk. This radial smoothing was carried out to ensure that the radius of the convective region is no less than the height of the disk. The local height of the disk is a natural choice for the smoothing radius, since $H$ is taken as the characteristic convection length when defining viscosity in (9).

The total viscosity coefficient was taken as the sum of the background and convective viscosities:

$$
\nu=\nu_{\mathrm{bg}}+\tilde{\nu}_{\mathrm{c}}
$$

Although this approach appears to overestimate the convective viscosity, we consider it to be an acceptable initial approximation. In future, we plan to apply mixing-length theory to carry out a more correct estimation of $\nu$ in convectively unstable regions. 


\subsubsection{Initial and boundary conditions, model for accretion from the envelope}

In the model, we use fixed values for the surface density at the inner $(R=0.1 \mathrm{AU})$ and outer $(R=100 \mathrm{AU})$ boundaries of the disk, $\Sigma=10^{-2} \mathrm{~g} / \mathrm{cm}^{2}$ and $\Sigma=10^{-5} \mathrm{~g} / \mathrm{cm}^{2}$, respectively. These boundary values of the surface density are three to four orders of magnitude lower than the values near these boundaries after the disk settles to a quasi-equilibrium state. We can assume that these boundary conditions enable the free flow of matter, i.e., that some effective mechanisms for the extraction of mass act at the boundaries of the disk. Such conditions certainly strongly affect the behavior of the solution near the boundary, as follows from the large surface density gradients near the boundaries, and they also determine the nature of the accumulation of gas in the disk. The introduction of more complex boundary conditions requires a separate study, which we plan to carry out in the future.

The evolution of the accretion disk is largely determined by the accretion of the matter from the circumstellar envelope. Simple estimates show that the star receives most of the matter from the disk, while the disk itself receives matter from the envelope, the remnant of the molecular cloud. The region of matter accretion from the envelope to the disk depends on the initial angular momentum of the cloud. To estimate the "centrifugal" radius $R_{\text {acc }}$, at which the matter from the envelope accretes, we used the formula from [23]

$$
R_{\mathrm{acc}}=\frac{\Omega^{2} R_{\mathrm{core}}^{4}}{G M_{\mathrm{core}}}
$$

where $\Omega$ is the angular velocity of the cloud, $R_{\text {core }}$ the initial position of the accreting element in the cloud, and $M_{\text {core }}$ the mass of the inner part of the cloud (the mass of the star). This formula is obtained from the condition of conservation of angular momentum of the accreting matter. The density distribution in observed protostellar clouds is usually described by the expression

$$
n=\frac{n_{0}}{1+\left(\frac{r}{r_{0}}\right)^{2}},
$$

where $n_{0}$ is the central number density of hydrogen and $r_{0}$ the radius of the inner region with almost constant density. One example of a well studied protostellar cloud is the prestellar core L1544, with $r_{0}=3 \times 10^{3}$ AU and $n_{0}=1.6 \times 10^{6} \mathrm{~cm}^{-3}$ [24]. Taking an accreting element located at the boundary of the plateau $\left(R_{\text {core }}=r_{0}\right)$, which in L1544 contains a considerable fraction of the cloud $\left(M_{\text {core }}=1.2 M_{\odot}\right)$, and assuming an angular velocity of $8.23 \times 10^{-14} \mathrm{~s}^{-1}$, 
corresponding to data for L1544 [25], the centrifugal radius $R_{\text {acc }}$ will be 11.3 AU. Obviously, more distant elements of the cloud should accrete at larger centrifugal radii. In our model, the accretion flow from the envelope onto the disk $W(R, t)$ is specified approximately, namely, we assumed a constant influx of matter into a ring between 10 and $20 \mathrm{AU}$ at the rate $10^{-7} M_{\odot} / \mathrm{yr}$. We chose a constant matter-influx rate because we considered only the initial stages of the disk evolution. At the initial time, we specified an initial distribution of the surface density $\Sigma(R) \propto R^{-1}$ and mass $10^{-7} M_{\odot}$ of a disk around a solar-mass star. This initial disk has only a nominal, formal nature, since the mass of the disk after reaching a quasi-equilibrium state will significantly exceed this value. In fact, in our formulation of the problem, the protoplanetary disk is formed completely via the accretion of gas from the envelope.

\subsubsection{Method used to solve the Pringle equation}

We solved the Pringle equation using a finite-difference method. The computational domain was split into cells, and Eq. (1) was approximated in a fully implicit form:

$$
\begin{aligned}
\frac{\Sigma_{i}^{(n+1)}-\Sigma_{i}^{n}}{\Delta t}= & \frac{3 r_{i+1}^{1 / 2}}{R_{i} \Delta r_{i} \Delta R_{i+1}}\left(\nu_{i+1} R_{i+1}^{1 / 2} \Sigma_{i+1}^{(n+1)}-\nu_{i} R_{i}^{1 / 2} \Sigma_{i}^{(n+1)}\right) \\
& -\frac{3 r_{i}^{1 / 2}}{R_{i} \Delta r_{i} \Delta R_{i}}\left(\nu_{i} R_{i}^{1 / 2} \Sigma_{i}^{(n+1)}-\nu_{i-1} R_{i-1}^{1 / 2} \Sigma_{i-1}^{(n+1)}\right),
\end{aligned}
$$

where $\Sigma_{i}^{(n)}$ is the surface density in cell $i$ for time step $n, \Delta t$ the time step, $r_{i}$ the left-hand border of cell $i, R_{i}$ the center of cell $i, \Delta r_{i}$ the linear scale of cell $i$, and $\Delta R_{i}$ the distance between the centers of cells $i$ and $(i-1)$ :

$$
\begin{aligned}
& R_{i}=\frac{1}{2}\left(r_{i}+r_{i+1}\right) \\
& \Delta r_{i}=\left(r_{i+1}-r_{i}\right) \\
& \Delta R_{i}=\left(R_{i}-R_{i-1}\right) .
\end{aligned}
$$

Equations (15) form a system of linear algebraic equations (with respect to the unknowns $\left.\Sigma_{i}^{(n+1)}\right)$ of the form:

$$
A_{i} \Sigma_{i-1}^{(n+1)}+B_{i} \Sigma_{i}^{(n+1)}+C_{i} \Sigma_{i+1}^{(n+1)}=D_{i}
$$

Together with the equations realizing the boundary conditions, these equations form a system of linear algebraic equations with a tridiagonal matrix. The solution of this system was found using the tridiagonal matrix method. The use of an implicit scheme to approximate 
the initial equation enabled us to make a reasonable choice of the time step based on the adopted accuracy, since this method is absolutely stable. In our computations, the time step was selected based on the rate of variation of the surface density. In particular, the time step was decreased when convectively unstable regions were identified.

\subsection{Vertical Structure of the Disk}

To compute the vertical structure of the disk, we used the thermal model of [26]. In this model, the one-dimensional radiative-transfer problem in the vertical (polar) direction is solved, taking into account heating by internal sources and external radiation, as well as the transfer of thermal radiation in the disk itself. The model assumes that the only source of opacity is dust, and that the temperatures of the gas and dust are equal. The dust-to-gas ratio was assumed to be constant throughout the disk and equal to $10^{-2}$. To solve this problem, the radiation was separated into ultraviolet (stellar and interstellar) and infrared (thermal) radiation of the disk itself. The intensity of the UV radiation was found via direct integration of the radiative-transfer equation. The heating of the disk by the UV radiation of the star can be found from the formula:

$$
S_{\mathrm{star}}=\frac{\kappa_{\mathrm{P}}^{\mathrm{uv}} L}{4 \pi R^{2}} \exp \left(-\tau_{\mathrm{uv}} / \cos \theta\right)
$$

where $\kappa_{\mathrm{P}}^{\mathrm{uv}}$ is the Planck-averaged absorption coefficient for the temperature of the star, $L$ the stellar luminosity, $\tau_{\text {uv }}$ the optical depth to UV radiation in the vertical direction from a given position to the upper boundary of the disk, and $\cos \theta \operatorname{cosine}$ of the angle between the normal and the direction toward the star. The luminosity of the star was taken equal to be the sum of the photospheric and accretion luminosities. The luminosity of the photosphere was assumed to be equal to the solar luminosity, and the accretion luminosity was calculated from the rate of accretion of matter from the disk onto the star. When computing $\kappa_{\mathrm{P}}^{\mathrm{uv}}$ and $\tau_{\mathrm{uv}}$, we used the stellar radiation temperature $T_{\mathrm{star}}=6000 \mathrm{~K}$. The quantity $\cos \theta$ was specified to be constant over the disk and equal to 0.05. This approximation substantially

simplifies the computation of the external heating, but it does not describe the effects of self-shadowing that can arise if the density is distributed non-monotonically. The heating by interstellar UV radiation was calculated using the formula:

$$
S_{\mathrm{bg}}=D \kappa_{\mathrm{P}}^{\mathrm{uv}} \sigma T_{\mathrm{bg}}^{4} \exp \left(-2 \tau_{\mathrm{uv}}\right)
$$


where $T_{\mathrm{bg}}=10^{4} \mathrm{~K}$ and $D=10^{-14}$ are the temperature and dilution of the interstellar radiation and $\sigma$ is the Stefan-Boltzmann constant. Note that the contribution of heating by interstellar radiation is insignificant for the regions under consideration, compared to heating by the central star, and can therefore be neglected.

To simulate the transfer of thermal radiation, a system of moment equations was solved in the Eddington approximation, using frequency-averaged absorption coefficients:

$$
\begin{aligned}
& c_{\mathrm{V}} \frac{\partial T}{\partial t}=\kappa_{\mathrm{P}} c\left(E-a T^{4}\right)+S \\
& \frac{\partial E}{\partial t}-\frac{\partial}{\partial z}\left(\frac{c}{3 \rho \kappa_{\mathrm{R}}} \frac{\partial E}{\partial z}\right)=-\rho \kappa_{\mathrm{P}} c\left(E-a T^{4}\right),
\end{aligned}
$$

where $T$ is the temperature of the medium, $E$ the radiative energy density, $z$ the vertical coordinate, $\rho$ the volumetric density, $c_{V}$ the specific heat of the medium, $c$ the speed of light, a Stefan's constant, $\kappa_{\mathrm{P}}$ the Planck-mean opacity, $\kappa_{\mathrm{R}}$ the Rosseland-mean opacity, and $S$ the heating function (per unit mass) for heating by stellar and interstellar radiation, as well as viscous friction:

$$
S=S_{\mathrm{star}}+S_{\mathrm{bg}}+\frac{\Gamma_{\mathrm{vis}}}{\Sigma}
$$

Despite the fact that the initial thermal model is non-stationary, the solution of the system (19)-(20) was found in a steady-state approximation. In other words, it was assumed that the time to reach thermal equilibrium in the vertical direction is significantly lower than the timescale for the viscous evolution at the given radius. Our test computations and estimates, similar to those presented in [27], showed that this approximation works well for the adopted model. However, for other parameters of the model, the steady-state condition could be violated in convectively unstable regions, where the characteristic time to reach thermal equilibrium and the viscous-evolution timescale can be comparable. Therefore, in the general case, a non-stationary model should be used. However, the use of a non-stationary thermal model will also require the computation of the convective heat transfer in the radial direction, which is beyond the scope of the $1+1 \mathrm{D}$ approach used here.

An important feature of the thermal model is the use of the temperature-dependent Planck-mean and Rosseland-mean opacities. These opacities were computed from the frequency-dependent absorption and scattering coefficients for a mixture of graphite and silicate dust particles. The spectral absorption and scattering coefficients themselves were computed using Mie theory, taking size distribution of dust particles to be a power law, $n(a) \propto$ $a^{-3.5}$, with the minimum and maximum radii of the dust particles $a_{\text {min }}=5 \times 10^{-7} \mathrm{~cm}$ and 

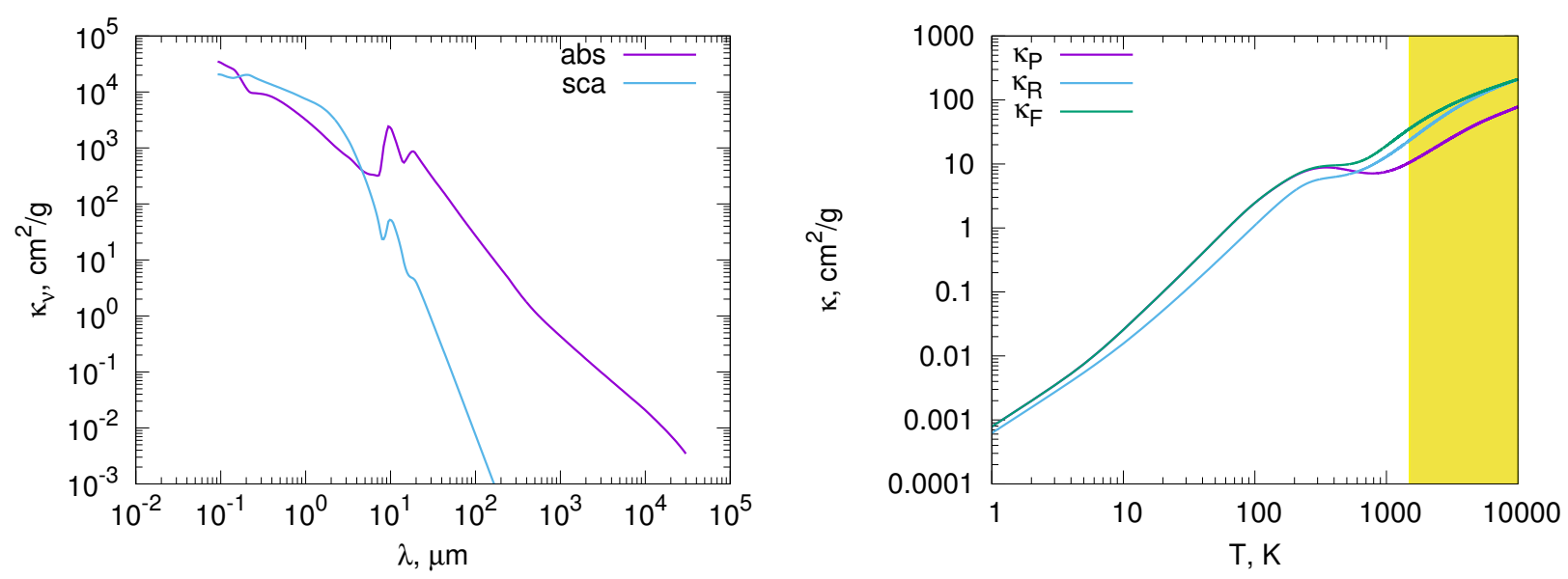

Figure 1. Wavelength dependence of absorption and scattering coefficients for a mixture of silicate and graphite dust particles (left panel) and the corresponding Plank-mean $\kappa_{\mathrm{P}}$, Rosseland-mean $\kappa_{\mathrm{R}}$ and flux-mean $\kappa_{\mathrm{F}}$ absorption coefficients (right panel).

$a_{\max }=10^{-4} \mathrm{~cm}$. The wavelength-dependent and corresponding Planck-mean, Rosselandmean, and external flux-averaged opacities are shown in Fig. 1. An important feature of the mean opacities is their growth with temperature at $T>500 \mathrm{~K}$. As our computations show, this is one of the conditions for the appearance of convectively unstable regions.

Note, our models do not account for evaporation of the dust at $T \gtrsim 1500 \mathrm{~K}$, when there should be a sharp decrease in opacity. At such high temperatures, the gas starts to provide the main input to the opacity. Moreover, at $T \gtrsim 2000 \mathrm{~K}$, dissociation and ionization of the hydrogen become substantial, but they are likewise not included in the model. In Fig. 1, the temperature range where the opacities we have used are, strictly speaking, incorrect, is marked in yellow. In our models, the temperature of the medium is usually below this critical range, but it can approach these values at the maximum temperature, leading to the need to modify the model. Such modifications will significantly complicate the thermal model, and we plan to carry out such a modification in the future.

The solution of the system of equations (19)-(20) was determined using a fully implicit scheme, which makes the method absolutely stable and removes restrictions on the time step. This method enabled us to correctly compute the thermal evolution in all regions of the disk, including optically thick regions, where the characteristic heating and cooling timescales are comparable to the dynamic timescales. The method used to model the thermal structure is closely related to the method used to reconstruct the vertical structure of the disk assuming 
local hydrostatic equilibrium, which was found from the equation:

$$
\frac{k_{B}}{\mu_{\mathrm{g}} m_{\mathrm{H}}} \frac{d(\rho T)}{\rho d z}=-\frac{G M_{*}}{r^{3}} z-4 \pi G \sigma,
$$

where $M_{*}$ is the stellar mass and $\sigma$ the surface density measured in the direction from the equator. The first term on the right-hand side of (22) takes into account the vertical component of the stellar gravitational field, and the second term takes into account the selfgravity of the disk. Note that, with the model parameters used, the self-gravity of the disk can be neglected. The computation of the vertical structure of the disk enabled us to obtain complete information about the distribution of the density and temperature in the disk. We also used a stable implicit method to solve the equation of hydrostatic equilibrium equation.

The fundamental condition for the efficiency of these methods is optimal choice of the spatial grid in the $z$ direction. The spatial grid must track all previously unknown features of the solution (the density and temperature gradients), taking into account the existence of a significant restriction on the number of cells (no more than 100) and the large intervals of the gas density (up to 10 orders of magnitude). We have developed an algorithm for the construction and adaptive modification of such a grid, based on an approximate fast solution of the equation of hydrostatic equilibrium. This method for the reconstruction of the vertical structure of the disk taking into account radiative transfer was thoroughly tested and compared with other methods. In the stationary regime, the temperature distributions are in good agreement with the results of modeling of the disk structure obtained in other studies. In the non-stationary regime, the characteristic times for establishing thermal equilibrium correspond to analytical estimates. A more detailed description of this method can be found in $[26]$.

\section{RESULTS OF THE MODELING}

We will now consider the simulation results for $35000 \mathrm{yr}$ after the onset of the evolution of the adopted disk model. Starting from this time, a periodic, burst-like character of the accretion is established: the inner region of the disk $(R<3 \mathrm{AU})$ is gradually filled with matter, followed by a relatively rapid transfer of matter from the inner region of the disk to the star due to convection, after which the process is repeated. Further, this time will be arbitrarily taken to be the zero point in time. Fig. 2 shows the evolution of the distributions 

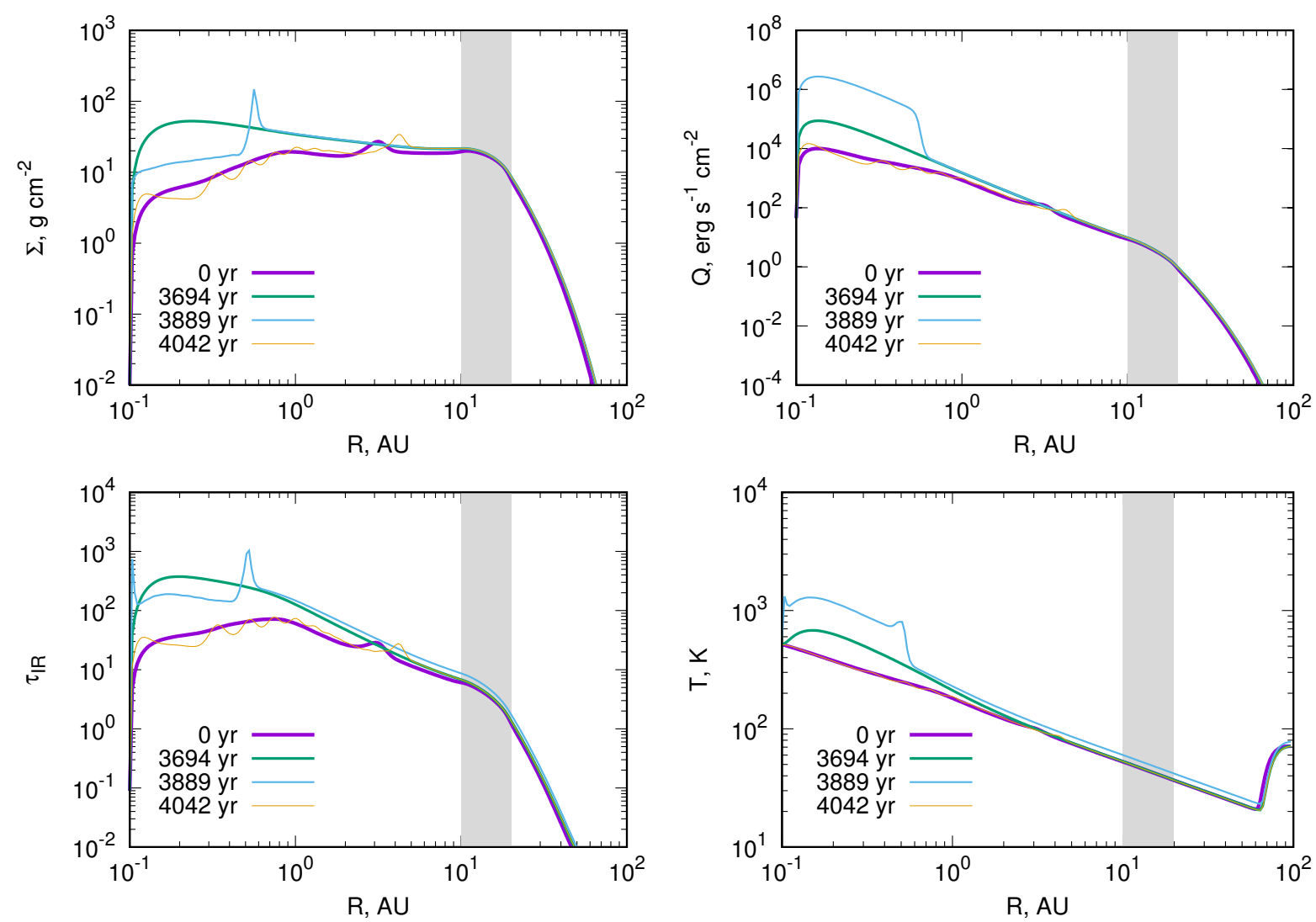

Figure 2. Radial distributions of the surface density (upper left panel), viscous heating rate (upper right panel), optical depth in the infrared (lower left panel), and equatorial temperature (lower right panel) for several times, illustrating the development of an accretion burst. Time is measured from the end of the previous accretion burst. The vertical strip shows the region of gas accretion from the envelope.

of the gas surface density, the rate of viscous heating, the optical depth to infrared radiation, and the temperature during an ordinary cycle of this burst accretion regime.

At the initial (zero) time, the surface density in the inner region $R<3$ AU increases in the radial direction from the star as a consequence of the transfer of matter after the previous cycle. The equatorial temperature of the disk monotonically decreases from the inner edge of the disk to $R \approx 60 \mathrm{AU}$, then experiences a jump. This jump at the outer boundary is due to the fact that the surface density is so small that the disk becomes transparent to the UV radiation of the star in the vertical direction; i.e., in the one-dimensional approximation applied, the equatorial regions are heated by the stellar radiation directly.

The inner region $(R<3 \mathrm{AU})$ is gradually filled with the accreted matter. At $t=3694 \mathrm{yr}$, 
the density distribution in the inner region becomes more monotonic. The temperature in the inner region increases. This is associated with an increase in the surface density and an accretion flow in the region. The optical depth in the infrared increases to $\tau_{\text {IR }} \approx 400$ at the radius $R=0.2 \mathrm{AU}$.

At this point, the inner region becomes convectively unstable, the region of convective instability propagates outward, and a density maximum forms at its front. At time $t=$ $3889 \mathrm{yr}$, the front reaches the radial distance $0.5 \mathrm{AU}$. The energy release $Q$ inside a radius of $0.5 \mathrm{AU}$ at $t=3889 \mathrm{yr}$ is approximately two orders of magnitude higher than it was before the formation of the convectively unstable zone (Fig. 2). The temperature inside the convection zone at this time increases substantially, reaching $1000 \mathrm{~K}$ at a radius of $0.2 \mathrm{AU}$. Beyond the boundary of the convective region, the temperature also rises compared to the temperature characteristic for the previous time period. This is a consequence of the addition to the stellar photospheric luminosity of significant heat energy due to the enhanced accretion of the disk material by the star. At $t=4042 \mathrm{yr}$, the expanding convective front attains the radius $R=3 \mathrm{AU}$. The surface density inside this radius decreases significantly, compared to the time before the outburst, while the density distribution itself becomes close to the initial distribution for time zero, with the difference that weak oscillations are visible in the distribution, which are later smoothed out. As Fig. 2 shows, at $t=4042$ yr, the other distributions are also close to the corresponding distributions at time zero. From this moment on, the inner region becomes stable to convection and the disk enters a new stage of matter accumulation.

Figure 3 shows the density and temperature distributions in the polar cross sections of the disk for three times, illustrating the development of an accretion outburst. The panels in the upper row of Fig. 3 show the distributions for the zero time (the beginning of the cycle). The gas density in the entire disk decreases monotonically from the equator to the upper boundary of the disk. Near the inner boundary of the disk, the density contrast of the disk in the polar direction reaches $\sim 11$ orders of magnitude, falling from $10^{14}$ at the equator to $10^{3} \mathrm{~cm}^{-3}$ at the upper boundary. At the same time, the character of the temperature distribution is defined by the distance from the star. At $R \gtrsim 1 \mathrm{AU}$, the temperature grows monotonically from the equator to the disk atmosphere, while, at $R \lesssim 1 \mathrm{AU}$, the temperature first falls off, then grows with increasing $z$. This is related to the circumstance that, in the inner region of the disk, viscous dissipation begins to provide a substantial input to the 

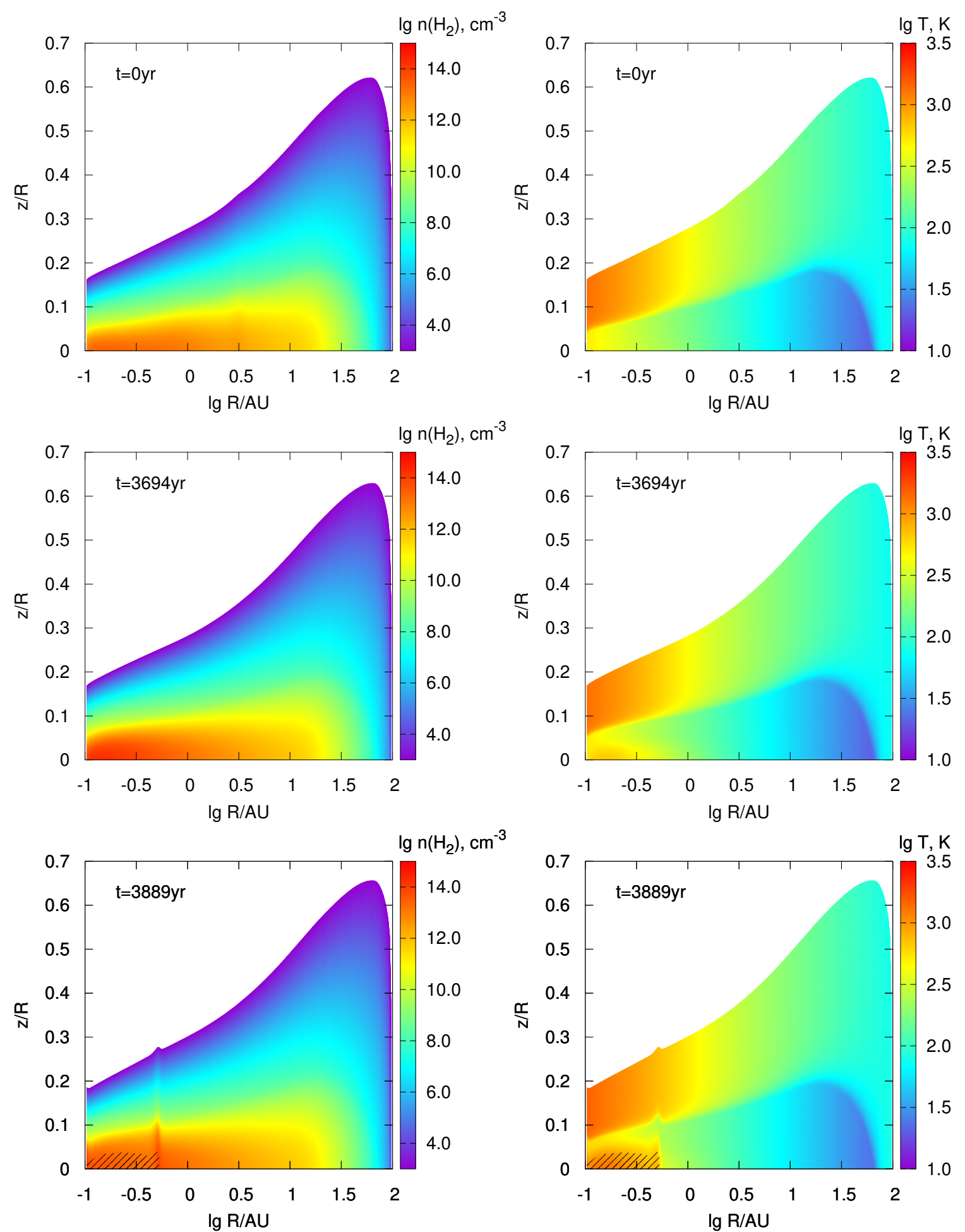

Figure 3. Distributions of the gas number density (left panels) and gas temperature (right panels) in an RZ cross section of the disk for three times illustrating the development of an accretion burst. The time is measured from the end of the previous accretion burst and is indicated in the upper left corner of each panel. The vertical axis shows the ratio of the height above the equator to the radial distance to the star. Convectively unstable regions are shaded. 
heating of the disk, while, at $R \gtrsim 1 \mathrm{AU}$, the dominant source of heating is the UV radiation of the central star.

The panels in the middle row of Fig. 3 show the distributions of the density and temperature at $t=3694 \mathrm{yr}$, corresponding to the time just before the enhancement of the accretion due to convection. Comparing these distributions with the upper panels for $t=0$, we note that the number density (up to $10^{15} \mathrm{~cm}^{-3}$ ) and the temperature of the gas at the equator (up to $10^{3} \mathrm{~K}$ ) have grown substantially in the inner region of the disk, $R<3 \mathrm{AU}$. This is related to the accumulation of matter in this region as a result of accretion from outer regions of the disk. The density and temperature distributions in the vicinity of $\lg R($ a.e. $)=0.5$ become more monotonic compared to these distributions at the initial time.

The distributions in the lower panels of Fig. 3 are for $t=3889 \mathrm{yr}$ and correspond to the phase of developed convection and the transfer of matter from the inner region. In the distribution of density in the vicinity of $\lg R=-0.3$, a region of enhanced density is seen, corresponding to the convection propagation front. The thickness of the disk inside this region is slightly greater than in the stage of matter accumulation, due to the increased temperature in the convection zone. In all the distributions, the shaded areas indicate the regions that satisfy the criterion of convective instability. The region of convective instability is located near the equator and extends to the height $z / R \approx 0.03$.

Figure 4 shows the distributions of the ratio of the temperature gradient $d T / d z$ and the absolute value of the adiabatic gradient $g(z) / c_{p}$ in the $z$ direction for two radii at the stage of the burst in accretion $(t=3889 \mathrm{yr})$. In the distribution for $R \approx 0.2 \mathrm{AU}$ (the left panel of Fig. 4), there is a region where this ratio drops below -1 , for which the condition of convective instability is satisfied. At $R=1 \mathrm{AU}$ (the right panel of Fig. 4), there is also a negative temperature gradient in the vicinity of the equator, but its absolute value does not exceed the value of the adiabatic gradient. Therefore, this region remains convectively stable at the given time.

Figure 5 shows the dependences of the accretion flux on the radius for the times just after the previous accretion burst $(t=0 \mathrm{yr})$, before the current burst (3694 yr), and in the phase of matter transfer (3889 yr).

In the phase of matter accumulation $(0<t<3694 \mathrm{yr})$, the disk can conveniently be divided into two parts, corresponding to accretion and decretion (using the terminology from [28]). In the accretion part, $R<15 \mathrm{AU}$, the matter moves toward the star, while the 

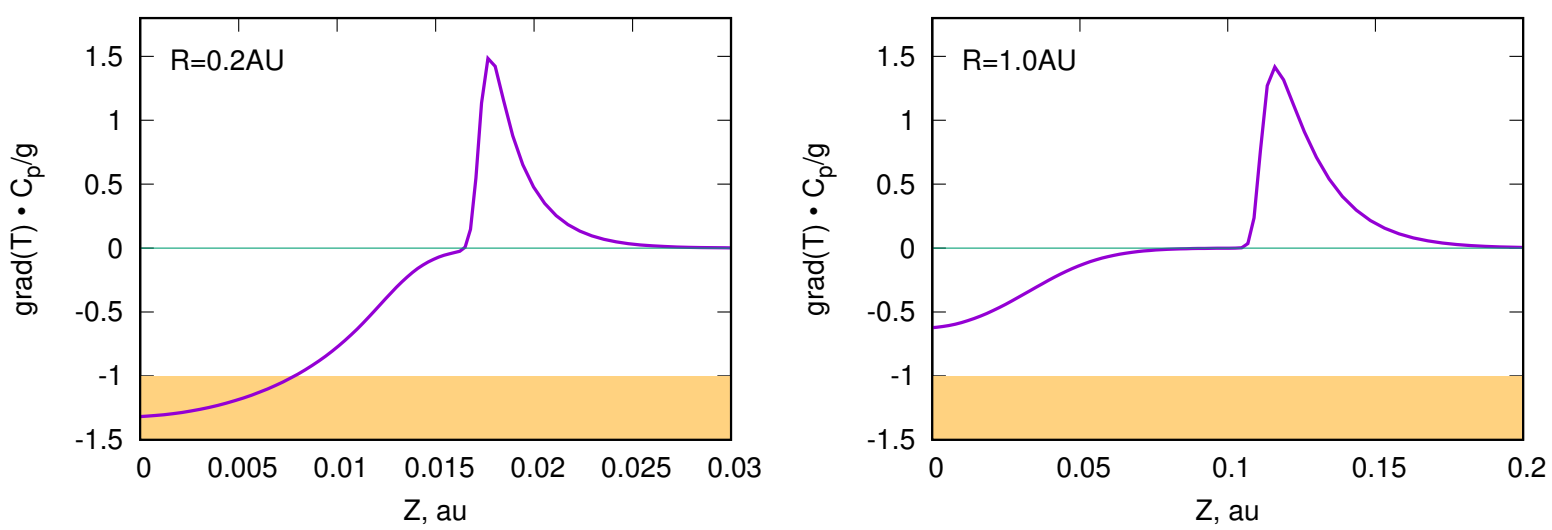

Figure 4. Ratio of the temperature gradient and adiabatic gradient as a function of the $z$ coordinate for $R=0.2 \mathrm{AU}$ (left) and $R=1 \mathrm{AU}$ (right) for time $3889 \mathrm{yr}$, corresponding to the phase of an accretion burst. The orange strip corresponds to the region of convective instability.

gas moves outward in the decretion part, $R>15 \mathrm{AU}$. In our model, the border between these regions is defined by the continuous influx of matter from the envelope into the ring at 10-20 AU. The absolute values of these fluxes on both sides of this border are close to $0.5 \times 10^{-7} M_{\odot} / y r$, i.e., to half the accretion rate from the envelope. Thus, about half of the mass of the accreting envelope enters the inner part of the disk, while the other half goes into the outer part. Note, however, that, in the model presented, the matter accreted from the envelope is fed into the disk at the Keplerian speed, and with other assumptions, this picture will change. In general, this boundary can shift as the disk evolves. At time $3694 \mathrm{yr}$, the flow of matter in the accretion part of the disk decreases toward the star, taking its minimum value at the inner boundary of the disk, thus providing evidence for the accumulation of matter in this region.

In the phase of the accretion burst (lower panel of Fig. 5), a characteristic feature is seen in the flux distribution - a peak of positive flux with amplitude $5 \times 10^{-7} M_{\odot} /$ yr near a radius of $0.5 \mathrm{AU}$; this peak corresponds to the front of the convective region. As was noted above, this front propagates outward. Inside a radius of $0.5 \mathrm{AU}$, the accretion flux exceeds the values characteristic for the accumulation phase by more than an order of magnitude. At the same time, the flux distribution upstream of the convective front, i.e., at $R>0.5 \mathrm{AU}$, remained the same.

Figure 6 shows the rate of accretion onto the star (left panel) and the accretion luminosity (middle panel) for a time interval of $12000 \mathrm{yr}$ after the establishment of the episodic accretion 

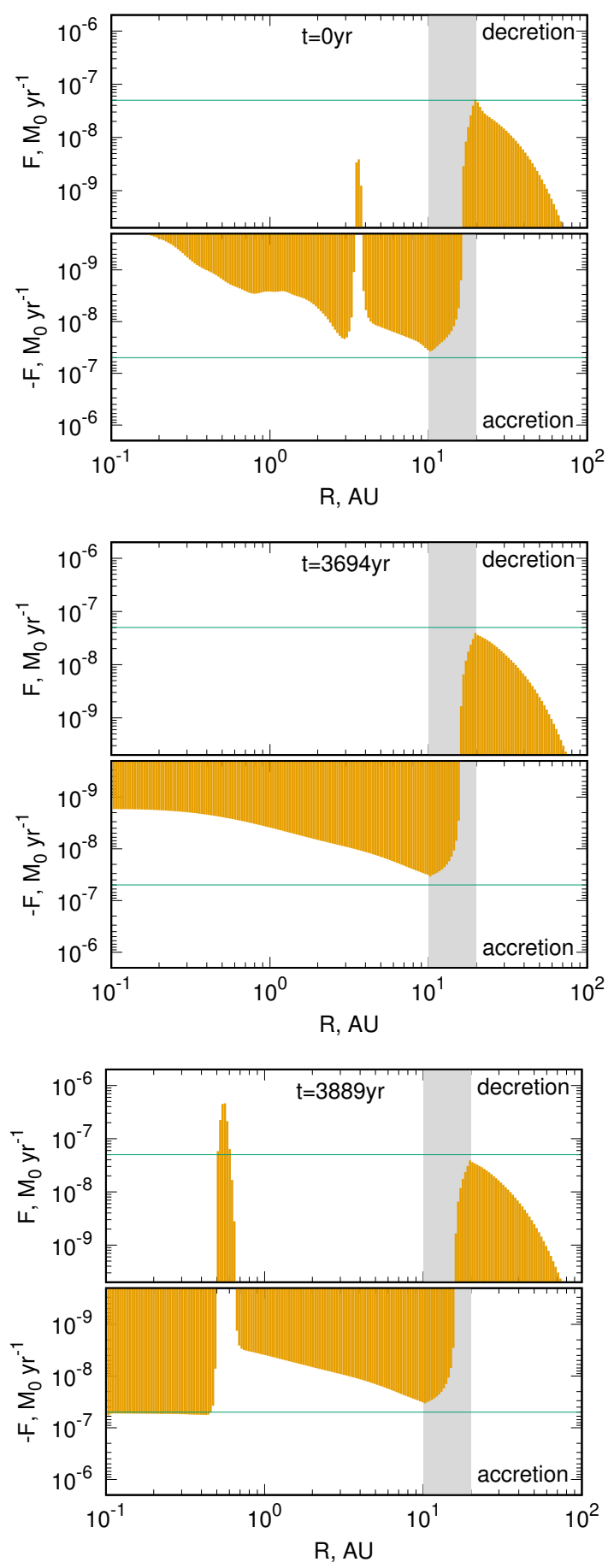

Figure 5. Radial distributions of the accretion flux at time zero (upper panel), just before the burst (middle panel), and during the burst (lower panel). Positive fluxes (the upper part of every distribution) correspond to flows from the star, and negative fluxes (the lower part of the distribution) to flow toward the star. The time is measured from the end of the previous accretion burst and is indicated at the top of every panel. Horizontal lines correspond to fluxes $\pm 0.5 \times 10^{-7} M_{\odot} /$ yr. The vertical strip indicates the region of accretion onto the star. 

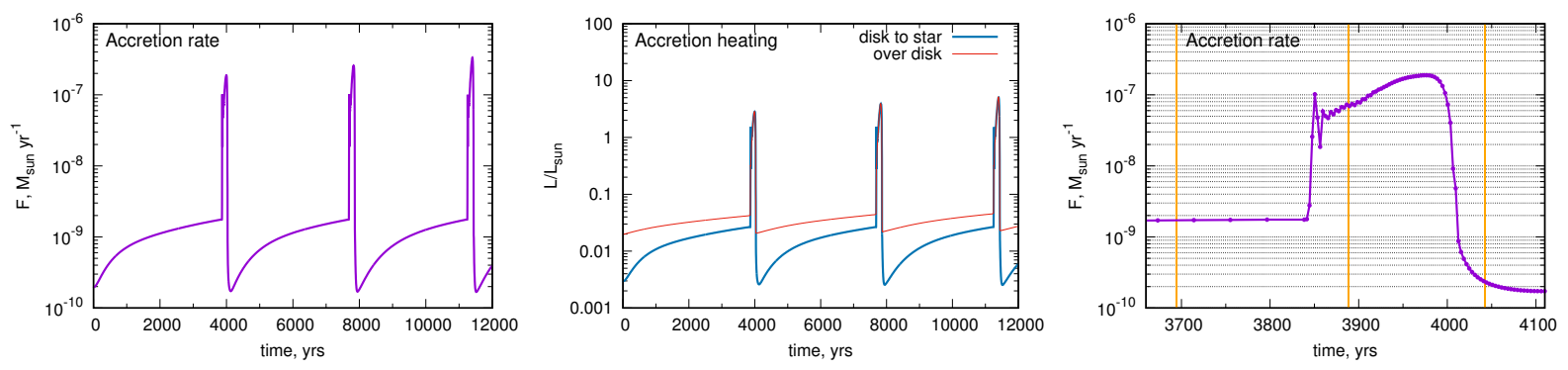

Figure 6. Dependence of the rate of accretion of the disk gas by the star (left panel) and the accretion luminosity (central panel) for a time $12000 \mathrm{yr}$ after the establishment of the episodic accretion regime. The rate of accretion onto the star during the burst phase is shown in the right panel. The red curve in the middle panel shows the accretion luminosity of the entire disk, the blue curve shows the luminosity associated with the accretion of gas from the inner boundary of the disk onto the star. The vertical (orange) lines in the bottom panel correspond to times of 3694,3889 , and 4042 yr.

regime, as well as the rate of accretion onto the star during the accretion burst phase (right panel). The accretion luminosity of the entire disk (red curve in Fig. 6) was computed by integrating expression (2) from the inner to the outer boundary of the disk. The accretion luminosity associated with the accretion of gas from the inner boundary of the disk onto the star was computed using the formula

$$
L_{*}=\frac{1}{2} \frac{G M_{\odot} \dot{M}}{R_{\odot}},
$$

where $M_{\odot}$ and $R_{\odot}$ are the mass and radius of the star (assumed to be the solar values). The dependences given above show that the interval between accretion outbursts is about 4000 yr, while the convective phase itself lasts about 200 years. The rate of accretion onto the star during the convective phase is approximately two orders of magnitude higher than the rate of accretion in the quiescent period. The total rate of energy release in the disk itself and at the surface of the star during the active phase is about $6 L_{\odot}, 50-100$ times higher than the values characteristic for the phase of gas accumulation in the disk.

\section{DISCUSSION}

Comparing the model rates of accretion and luminosity with those for young bursting FU Ori (FUor) and EX Lup (EXor) objects [29], we can conclude that our model reproduces 
the characteristic duration of FUor bursts (from several tens to hundreds of years) fairly well. It is difficult to derive the duration of the quiescent phase between the bursts of FUors from observations; it varies from several thousand years [30] to several tens of thousands of years [31]. In our model, it is close to the lower limit of observational estimates. On the other hand, the maximum accretion rate during a burst is close to the lower limit of observational estimates and is consistent only with data for NGC 722 [29]. With regard to the maximum accretion rate and accretion luminosity, our model is more consistent with EXor-type bursts, but these objects have much shorter and more frequent bursts lasting from several months to several years [29]. Note that we have not yet studied the entire range of possible model parameters. In particular, we have not investigated the dependence of the disk evolution on the law for gas accretion from the envelope onto the disk. Test computations show that with an increase in the rate of accretion from the envelope, the time between bursts decreases, but the duration and intensity of the bursts are preserved. Further investigation of the episodic accretion regime caused by convective instability is needed for a more detailed comparison with observations.

Note that our model is largely illustrative due to the numerous physical approximations applied. Its main purpose is to describe the evolution of the disk, and to show the possible role of convection in enabling an episodic accretion regime. This picture is presented schematically in Fig. 7.

We will now list a number of important issues that underlie the model and require special attention in its further development. We have assumed that, along with convection, another unspecified mechanism acts in the disk, which provides a "background" viscosity. It is due to this mechanism that the transfer of mass and angular momentum is realized in the quiescent phase of the disk's evolution. Due to the background viscosity, matter accumulates in the inner region of the disk. In our model, convection is a trigger that facilitates the transfer of matter from the inner region of the disk.

In our model, a fundamental condition for the appearance of convectively unstable regions is an increase in the opacity of the medium with increasing temperature (see Fig. 1). This is brought about by the optical properties of the dust used in the model, namely, the increase of the spectral absorption and scattering coefficients with frequency. Our test computations show that, when using artificially specified opacities that are independent of temperature, the resulting temperature gradient is not strong enough to ensure condition (8). The strong 

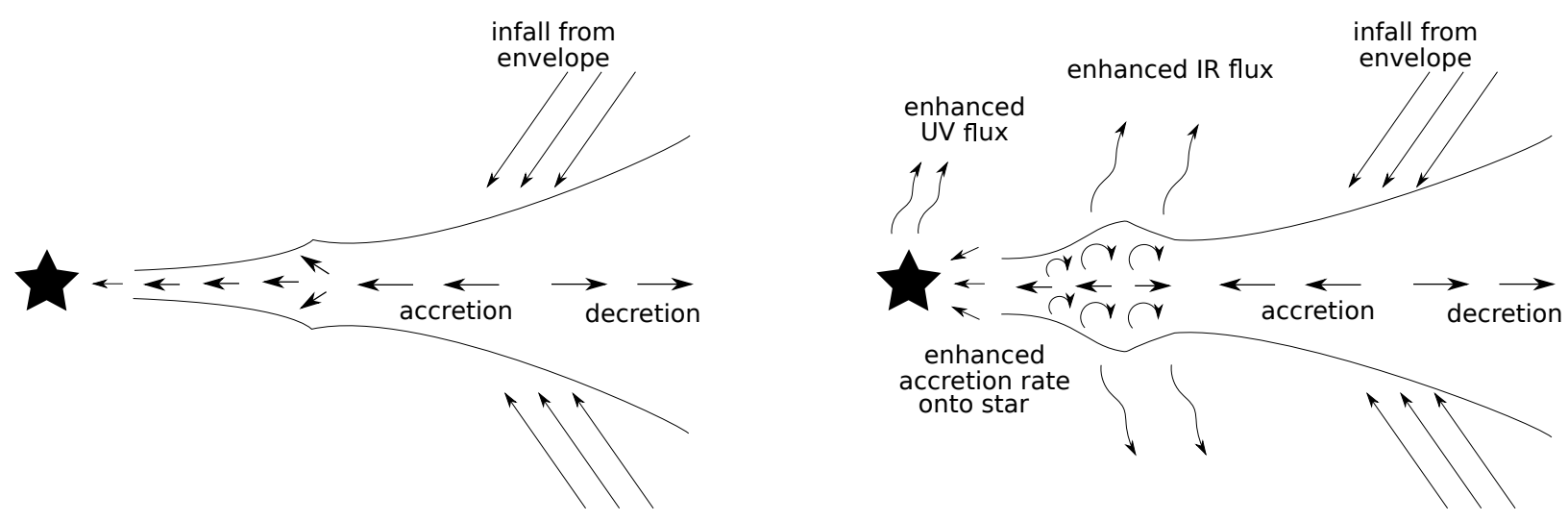

Figure 7. Sketch illustrating the burst-like nature of accretion in protoplanetary disks. The matter-accumulation phase is shown to the left and the convective phase to the right.

influence of the opacity law on the appearance of convectively unstable zones was noted in [32, 33], where the structures of protoplanetary disks with various parameters were calculated and analyzed. It was shown, in particular, that the evaporation of dust in the inner regions of the disk reduces the opacity of the medium, eliminating the appearance of convectively unstable regions. In our model, dust evaporation is not taken into account, but the maximum temperature can approach the characteristic dust evaporation temperatures. Therefore we plan to take this process into consideration in the future.

In our model, an increase in the opacity with increasing temperature also leads to positive feedback in the development of convection in the inner region of the disk: an increase in the accretion rate leads to an increased energy release, an increased temperature, and, as a result, to more favorable conditions for the appearance of convective instability. However, the convectively unstable region is depleted relatively quickly (in about hundred years). A strong decrease in the density leads to a decrease in the accretion rate and energy release, which ultimately leads to the restoration of convective stability in the inner region. Thus, in this model, convection is self-sustaining only for short periods of time in the inner regions of the disk, and the background viscosity is important to ensure its launch.

In our model, a prerequisite for the quasi-periodic (burst) accretion regime is a steady inflow of matter into the disk from the envelope. In our current realization of the model, the accretion of gas from the envelope was assumed to be constant, and the accretion region was a ring between 10 and $20 \mathrm{AU}$. In reality, the accretion rate and the position of the region of accretion of matter from the envelope are time dependent, and this must be taken into 
account when constructing models aimed at a more consistent comparison of the model with observations. With a decreasing rate of accretion from the envelope and depletion of the disk, the inner regions become more transparent to their own thermal radiation, and the rate of viscous heating decreases. As a result of all this, the conditions for convective instability cease to be satisfied.

An important element of the model is the method used to compute the viscosity coefficient in the convectively unstable region. We used an approximation in which the velocities of convective elements are calculated from the condition that all the thermal energy released as a result of viscosity is transported by the convective flow. In this approach, we do not allow for the fact that some of the energy can be carried by radiation, i.e., the velocity of the convective elements is somewhat overestimated. At the same time, when reconstructing the disk structure in the polar direction, we did not take into account the convective energy transfer and radiative energy transfer in the radial direction; i.e., the temperature distribution was found by taking into account radiative transfer in the vertical direction only, in a steady-state approximation. In order to eliminate these inconsistencies, we plan in the future to apply mixing-length theory, which has successfully been used to take into account convection in computations of stellar structure (see, e.g., [34]).

The picture we have presented here is only one possible scenario for the episodic behavior of the accretion around young stars. A wide variety of models have already been proposed for FUors and EXors. For example a two-dimensional hydrodynamical model is presented in [35], in which the burst character of the accretion is provided by magnetorotational instability, while gravitational instability is responsible for the influx of the matter into the inner regions of the disk from its outer part. In [36], the bursts of FUors are explained by the infall of gravitationally bound fragments that form in the accretion disk and migrate toward the star. We note also that, in order to explain the observational manifestations of FUors and the dynamics of the circumstellar gas, the presence of an intense wind generated by the inner regions of the turbulent accretion disk is necessary [37]. In general, recurrent accretion activity is a common occurrence in accreting objects [38].

In conclusion, we note the need to reproduce the obtained episodic character of accretion in the framework of a hydrodynamical model. In a hydrodynamical model, the development of convective zones can be observed directly; i.e., there is no need for a phenomenological introduction of the viscosity coefficient in the convective region. In a hydrodynamic model, 
however, it is necessary to pay increased attention to the computation of the thermal structure of the disk and the selection of a suitable spatial grid, due to the fact that the density gradients from the equator to the disk atmosphere amount to many orders of magnitude.

\section{ACKNOWLEDGMENTS}

We thank the referee for valuable comments and constructive suggestions for improvement of the paper. We also thank A. B. Makalkin for useful discussions.

\section{FUNDING}

This project was supported by the Russian Foundation for Basic Research (project 1702-00644).

1. P. J. Armitage, Astrophysics of Planet Formation (2013).

2. A. G. W. Cameron, Moon and Planets 18, 5 (1978).

3. D. N. C. Lin and J. Papaloizou, Monthly Notices Roy. Astron. Soc. 191, 37 (1980).

4. H. Klahr, in Convection in Astrophysics, edited by F. Kupka, I. Roxburgh, and K. L. Chan (2007), vol. 239 of IAU Symposium, pp. 405-416.

5. W. Cabot, V. M. Canuto, O. Hubickyj, and J. B. Pollack, Icarus 69, 423 (1987).

6. W. Cabot, V. M. Canuto, O. Hubickyj, and J. B. Pollack, Icarus 69, 387 (1987).

7. H. H. Klahr, T. Henning, and W. Kley, Astrophys. J. 514, 325 (1999).

8. H. H. Klahr and P. Bodenheimer, Astrophys. J. 582, 869 (2003), astro-ph/0211629.

9. G. V. Lipunov and N. I. Shakura, Izvestia Rossiiskoi Academii Nauk, Seria fizicheskaya 67, 322 (2003).

10. S. Hirose, O. Blaes, J. H. Krolik, M. S. B. Coleman, and T. Sano, Astrophys. J. 787, 1 (2014), 1403.3096.

11. M. S. B. Coleman, I. Kotko, O. Blaes, J. P. Lasota, and S. Hirose, Monthly Notices Roy. Astron. Soc. 462, 3710 (2016), 1608.01321.

12. L. E. Held and H. N. Latter, Monthly Notices Roy. Astron. Soc. 480, 4797 (2018), 1808.00267.

13. N. Shakura and K. Postnov, Monthly Notices Roy. Astron. Soc. 448, 3707 (2015), 1502.01888. 
14. N. Shakura and K. Postnov, Monthly Notices Roy. Astron. Soc. 451, 3995 (2015), 1506.00526.

15. K. L. Malanchev, K. A. Postnov, and N. I. Shakura, Monthly Notices Roy. Astron. Soc. 464, 410 (2017), 1609.03799.

16. J. E. Pringle, Annual Review of Astronomy and Astrophysics 19, 137 (1981).

17. P. J. Armitage, Annual Rev. of Astron. and Astrophys. 49, 195 (2011), 1011.1496.

18. J. P. Williams and L. A. Cieza, Annual Rev. of Astron. and Astrophys. 49, 67 (2011), 1103.0556.

19. L. Hartmann, N. Calvet, E. Gullbring, and P. D'Alessio, Astrophys. J. 495, 385 (1998).

20. N. I. Shakura and R. A. Sunyaev, Astron. and Astrophys. 24, 337 (1973).

21. P. Cossins, G. Lodato, and C. J. Clarke, Monthly Notices Roy. Astron. Soc. 393, 1157 (2009), 0811.3629 .

22. L. D. Landau and E. M. Lifshitz, Fluid mechanics (1959).

23. C. P. Dullemond, A. Natta, and L. Testi, The Astrophysical Journal Letters 645, L69 (2006), astro-ph/0605336.

24. A. Chacón-Tanarro, J. E. Pineda, P. Caselli, L. Bizzocchi, R. A. Gutermuth, B. S. Mason, A. I. Gómez-Ruiz, J. Harju, M. Devlin, S. R. Dicker, et al., Astron. and Astrophys. 623, A118 (2019), 1901.02476.

25. J. Klapp, L. D. G. Sigalotti, M. Zavala, F. Peña-Polo, and J. Troconis, Astrophys. J. 780, 188 (2014).

26. E. I. Vorobyov and Y. N. Pavlyuchenkov, Astron. and Astrophys. 606, A5 (2017), 1706.00401.

27. E. I. Vorobyov, Y. N. Pavlyuchenkov, and P. Trinkl, Astronomy Reports 58, 522 (2014).

28. A. V. Tutukov and Y. N. Pavlyuchenkov, Astronomy Reports 48, 800 (2004).

29. M. Audard, P. Ábrahám, M. M. Dunham, J. D. Green, N. Grosso, K. Hamaguchi, J. H. Kastner, Á. Kóspál, G. Lodato, M. M. Romanova, et al., Protostars and Planets VI pp. 387-410 (2014), 1401.3368.

30. E. I. Vorobyov, V. G. Elbakyan, A. L. Plunkett, M. M. Dunham, M. Audard, M. Guedel, and O. Dionatos, Astron. and Astrophys. 613, A18 (2018), 1801.06707.

31. A. Scholz, D. Froebrich, and K. Wood, Monthly Notices Roy. Astron. Soc. 430, 2910 (2013), 1301.3152.

32. A. B. Makalkin and V. A. Dorofeeva, Astronomicheskii Vestnik 29, 99 (1995).

33. A. B. Makalkin and V. A. Dorofeeva, Solar System Research 30, 440 (1996).

34. C. J. Hansen, S. D. Kawaler, and V. Trimble, Stellar interiors : physical principles, structure, 
and evolution (2004).

35. Z. Zhu, L. Hartmann, C. Gammie, and J. C. McKinney, Astrophys. J. 701, 620 (2009), 0906.1595 .

36. E. I. Vorobyov and S. Basu, Astrophys. J. 650, 956 (2006), astro-ph/0607118.

37. K. Milliner, J. H. Matthews, K. S. Long, and L. Hartmann, Monthly Notices Roy. Astron. Soc. 483, 1663 (2019), 1811.12453.

38. A. V. Tutukov and Y. N. Pavlyuchenkov, Submitted to Astron. Rep. (2019).

Translated by L. Yungelson 